\title{
Los proyectos de aula en la formación inicial de profesores de matemáticas
}

The projects of classroom in the initial formation of teachers of mathematics

\author{
Ana Cecilia Medina Mariño
}

Universidad Pedagógica y Tecnológica de Colombia - Duitama. aceciliamedina@gmail.com

\section{Resumen}

Esta ponencia presenta el proceso y resultados de un estudio sobre el conocimiento acumulado en los informes de los Proyectos de Aula desarrollados por estudiantes de décimo semestre de la Lic. en Matemáticas y Estadística de la Universidad Pedagógica y Tecnológica de Colombia, Duitama. Se pretende exponer algunos avances y limitaciones en relación con los componentes pedagógicos e investigativo de la formación inicial de profesores de matemáticas. Tratamos con ello de aportar elementos de reflexión para incidir en el progreso del programa, la consolidación de líneas de investigación en este campo, y el mejoramiento continuo de la calidad de la educación.

\section{Palabras clave}

Formación inicial de profesores de matemáticas, investigación- acción en el aula, conocimiento profesional, profesor reflexivo.

\section{Abstract}

This report presents the process and results of to study on the knowledge accumulated in the reports of the Projects of Classroom developed by students of tenth semester of the Degree in Mathematics and Statistic of the Pedagogic and Technological University of Colombia, Duitama. It is sought to expose some advances and limitations in connection with the pedagogic and investigative components of the initial formation of teachers of mathematics. We ty with it to contribute reflection elements to impact in the progress of the program, the consolidation of investigation lines in this field, and the continuous improvement of the quality of the education.

\section{Keywords}

Teachers' of mathematics initial Formation, investigation - action in the classroom, professional knowledge, reflexive teacher.

\section{Introducción}

En las últimas décadas la comunidad científica de Educación Matemática está de acuerdo en aceptar que no hay educación de calidad, ni reformas educativas efectivas sin una formación adecuada de los profesores. Probablemente esta idea sea una de las causas por la que se ha intensificado la investigación en la formación de profesores de matemáticas, aryos resultados están suginiendo cambios profundos en los currículos de los programas de formación inicial y permanente del profesorado. (Blanco, 1998).

Atendiendo a avances investigativos sobre la formación inicial de educadores matemáticos (Azcárate, 1998; Blanco, 1998; Font 2005), el programa de Lic. en Matemáticas y Estadística de 
la UPTC Duitama implementó la asignatura "Proyecto Pedagógico Fase I" desde el año 2002, en la aral se desarrolla un proyecto de investigación en el aula, con el propósito de articular teoría y práctica docente, y promover la figura del profesor de matemáticas como investigador de su propia práctica, condición para la construcción y desarrollo del conocimiento profesional.

En esta asignatura los estudiantes elaboran un informe escito, producto de la experiencia pedagógica e investigativa. Para aproximamos a la reauperación y reconstrucción del conocimiento acumulado en estos informes, en torno a los componentes pedagógico e investigativo de la formación inicial de profesores, se utilizó la metodología del Estado de Arte desde la perspectiva hermenéutica.

El balance documental se realizó tomando como objeto de estudio cincuenta y seis informes presentados por los estudiantes entre el año 2003 y el primer semestre de 2007. El proceso llevado a cabo en el desarrollo de este estudio, así como los resultados, hallazgos y limitaciones encontradas en el análisis interpretativo se estructuran en cuatro secciones.

En la primera parte, se presenta la experiencia de formación inicial. Luego se señalan algunos referentes teóricos que orientaron y sirvieron de marco de referencia interpretativo y se describe la metodología utilizada. En el siguiente apartado, se presentan los resultados a manera de argumentos de sentido que dan una visión comprensiva del conocimiento pedagógico e investigativo acumulado. Por último, se encuentran las conclusiones y algunas reflexiones generadoras de nuevas búsquedas e innovaciones relativas a la formación inicial del profesorado de matemáticas.

\section{La experiencia de formación inicial}

El proyecto curricular de la Licenciatura en Matemáticas y Estadística de la UPTC, Duitama ha venido experimentado cambios acordes a la evolución de las concepciones sobre la formación de los profesores.

En la estructura curricular del programa se introdujo la asignatura Proyecto Pedagógico Fase I con el fin de contribuir a la formación de un educador matemático autónomo, reflexivo y crítico, mediante la integración del saber pedagógico, saber didáctico, el saber matemático y las competencias investigativas, de tal forma que le permita confrontar la teoría con la práctica y generar una actitud reflexiva y crítica, para promover la evolución permanente de su conocimiento profesional.

La experiencia consiste en el diseño, desarrollo de un proyecto de investigación en el aula mediante la metodología de la Investigación- Acción (Elliot, 1994), con el fin de ayudar a superar errores y dificultades en el aprendizaje de las matemáticas en estudiantes de educación Básica y Media del Municipio e Duitama (Colombia).

El proceso investigativo se desarrolla en cinco fases, que se van ampliando en forma de espiral:

La fase exploratoria- diagnóstica consiste en la planificación y elaboración de un diagnóstico sobre las dificultades y enrores en el aprendizaje de conceptos y procedimientos espećficos de las matemáticas.

En la fase de planificación, se revisan referentes teóricos, con el fin de planificar y fundamentar un Proyecto de investigación en el Aula que ayude a resolver el problema identificado en el diagnóstico.

La fase de acción-reflexión, consiste en la intervención en el aula mediante el desarrollo de las secuencias didácticas con un enfoque constructivista y la aplicación de instrumentos de 
investigación. Se proporcionan espacios de reflexión con el fin de buscar soluciones a problemas surgidos durante la práctica.

La fase de evaluación se realiza durante el desarrollo y el final del proyecto, mediante el proceso de sistematización, en el cual la "práctica educativa" se convierte en objeto de estudio a la luz de las teorías de Didáctica de las Matemáticas.

Por último, en la etapa de difusión se elabora un informe final escrito que recopila el proceso y los resultados de la investigación, y se socializa ante la comunidad universitaria en eventos académicos y en la red.

\section{Referentes teóricos}

En esta sección se exponen en forma sintética algunos de los referentes teóricos que sirvieron de soporte para el desarrollo del trabajo.

\section{Sobre Proyecto de Aula}

A pesar de las diferencias en las formas de concebir un Proyecto de Aula, no hay duda que sus propósitos y recursos apuntan hacia un trabajo pedagógico e investigativo que se realiza en el ámbito espeáfico del Aula. Así, en la asignatura Proyecto Pedagógico Fase I se concibe de la siguiente manera:

"El proyecto de Aula es un Proyecto pedagógico e investigativo, planeado sistemáticamente que permite organizar las dinámicas del Aula o interacciones entre profesor, estudiantes y Saber, con el fin de atender alguna situación problemática del Aula, generar conocimientos, optimizar las formas de adquisición del conocimiento, e incentivar la investigación tanto en el docente como en los educandos. Trata de solucionar problemas, dificultades, obstáculos de aprendizaje en los estudiantes y responde a los intereses $y$ necesidades del grupo. Articula teoría didáctica, práctica docente e investigación en educación". (Medina, 2006)

En este sentido, el proyecto de Aula tiene como funciones: cualificar las prácticas docentes, mejorar los procesos de enseñanza y aprendizaje, promover competencias investigativas en los futuros educadores matemáticos, organizar y evaluar en forma sistemática el trabajo de aula para hacerlo eficaz, realizar adaptaciones curriculares, aplicar metodologías innovadoras y desarrollar el pensamiento matemático del estudiante en un proceso autónomo e interactivo.

El profesor como profesional reflexivo

En los estudios de formación de profesores Shön $(1983,1992$, citado por Flores, 2004) ha remarcado las diferencias que existen entre la formación teórica que suele dar la universidad y las necesidades prácticas que tienen los profesionales. Para Shön, la reflexión en y sobre la acción son mecanismos que los docentes prácticos reflexivos usan para su desarrollo continuo.

Van Manen (1998, citado por Flores, 2004) distingue los siguientes tipos de reflexión según el momento en que se realiza y la intención de la misma:

$>$ Reflexión anticipativa (para la acción). Reflexión en la planificación de las dases.

$>$ Reflexión activa o interactiva que permite al profesor afrontar problemas que aparecen en la acción.

> La conciencia de la actuación constituye otro tipo de reflexión.

> La reflexión sobre los recuerdos (sobre la acción) le ayuda a dar sentido a las experiencias pasadas, y de esta forma conseguir perspectivas sobre el significado de esas experiencias. 
Al reflexionar, los profesores pueden profundizar sobre los problemas con diferentes expectativas, por lo que Van Manen (1977, citado por Flores, 2004) establece como niveles de reflexión: Racionalidad Técnica (Nivel empírico-analítico, Acción práctica (Nivel hermenéuticofenomenológico) y Reflexión crítica (Nivel Crítico- Teórico). Al respecto Flores, P. apunta que estos niveles permiten entender si un profesor evoluciona en su reflexión.

Conocimiento profesional del profesor de matemáticas

En este estudio se han utilizado como referentes algunos principios de la formación inicial de profesores de matemáticas sugeridos por Azcárate (1998):

Para la formación de profesores se considera necesaria articulación teoría- práctica desde el inicio del proceso de desarrollo profesional, es dear desde el comienzo de la formación inicial, y se apoya en dos ideas eje que orientan y dan sentido a las decisiones curriculares.

Se promueve la figura del profesor de matemáticas como investigador de su propia práctica; en este sentido, se considera la investigación como base de la formación del profesorado, al igual que se considera como base de la enseñanza. (Porlán 1993, citado por Azcárate, 1998).

El conocimiento profesional del profesor de matemáticas no puede ser transferido sino que es construido en forma individual, colectiva y progresivamente pasando por concepciones sucesivas. Se parte de la explicitación de las concepciones iniciales, las cuales se confrontan con teorías didácticas; y luego desde la teoría se planifican propuestas didácticas que se someten a la confrontación en la práctica.

\section{Proceso metodológico}

Esta es una investigación documental cuya metodología consistió en la construcción del Estado del Arte desde la perspectiva hermenéutica, ayyo objeto de estudio lo conforman cincuenta y seis informes de los proyectos de investigación en el Aula desarrollados entre el año 2003 y primer semestre de 2007.(Bohórquez, 2008). Un estado del arte es una investigación fundamentada en el conocimiento acumulado, a partir del cual se construye un "dialogo de saberes" que conduce a formular nuevas comprensiones, hipótesis y propuestas de acción sobre el fenómeno que se ha investigado.

En este enfoque el proceso de construcción teórica avanza mediante las siguientes fases (Cifuentes y otros, 1993):

> Descripción estadística del conocimiento acumulado en los 56 informes de los Proyectos Pedagógicos Fase I, tomando como referencia las perspectivas histórica, institucional, poblacional, temática, metodológica y teórica de la investigación.

$>$ Elaboración de argumentos interpretativos para analizar los argumentos descriptivos. Con el fin de facilitar el análisis se organizaron tres nuevas categorías: Dimensión contextual, Investigativa y Pedagógica.

$>$ En la fase de constitución de sentido se analiza cáticamente los argumentos descriptivos e interpretativos con el fin de determinar las fortalezas 0 debilidades que revelan los informes. 
Visión comprensiva del conocimiento acumulado en torno a lo pedagógico e investigativo

A continuación se expresa la comprensión sobre avances y limitaciones que revelan los informes de los proyectos pedagógicos Fase I y algunas implicaciones en la formación de los futuros educadores matemáticos.

\section{$>$ Los proyectos de Aula en el sector educativo de Duitama: cobertura e impacto}

Los proyectos de investigación en el aula aportan a la formación de un profesor autónomo ya qué que con ellos se promueve el desarrollo de capacidades profesionales que le permiten diseñar, experimentar y evaluar unidades y secuencias didácticas, reflexionando en y sobre la práctica, para descubrir, criticar y modificar los modelos y creencias que subyacen a la misma (Azcárate, 1998).

De otra parte, la razón de ser de los proyectos pedagógicos Fase I es su finalidad, que consistente en apoyar a los estudiantes de Básica Secundaria y Media que presentan dificultades en el aprendizaje de las matemáticas. Se ha encontrado que la mayoría de dificultades se presentan en estudiantes grados $7^{\circ}, 6^{\circ}$ y $8^{\circ}$ en relación con los sistemas de los números naturales, enteros y racionales.

Los proyectos han tenido impacto positivo en el Municipio de Duitama ya que durante cinco años se han atendido 2374 estudiantes de seis instituciones educativas oficiales, y aunque solo el $54.59 \%$ ha logrado culminar satisfactoriamente el proyecto, el beneficio se puede relacionar con los resultados de las pruebas SABER aplicadas en el año 2006, pues en el análisis global del estudio final a nivel nacional del Ministerio de Educación se encontró que de todas las entidades tenitoriales, Duitama obtuvo el promedio más alto en todas las áreas. (Mineducación, 2006).

\section{$>$ El proyecto pedagógico fase I y la formación de una actitud reflexiva}

En este estudio se comparte la hipótesis de Flores, P. (2004) de que el aprendizaje del estudiante para profesor está vinculado al desarrollo de una actitud reflexiva ante la resolución de problemas relacionados con la práctica de enseñar matemáticas.

Se observa que los informes revelan diferentes tipos de reflexión en tiempos y espacios específicos. Según la clasificación de Van Manen (1998) se refleja reflexión anticipativa (para la acción) en los procesos de planificación; Reflexión activa o interactiva que permite al profesor afrontar problemas que aparecen en la acción. La reflexión sobre la acción es la que realizan en forma individual y colectiva los estudiantes-profesores después de cada intervención en el aula y al final del proyecto. Todo esto, ayuda determinar debilidades y fortalezas para cualificar las prácticas docentes.

Al igual que la apreciación de Flores, P. (2004), consideramos que una de las limitaciones encontradas para desarrollar plenamente una actitud investigativa es el hecho de que la mayoría de acciones se realizan para responder a las exigencias académicas de la asignatura, y no porque la actitud reflexiva se genere en forma intencional por interés personal o profesional. Sin embargo, se espera que estas primeras experiencias de reflexión programada generen hábitos de reflexión sistemática, cuando se desempeñen como profesores totalmente autónomos.

\section{$>$ Tendencias teóricas que han orientado los Proyectos de Aula}

Existe una marcada influencia teórica de la Educación Matemática española. Tanto los diagnósticos sobre errores y dificultades en el aprendizaje de las matemáticas como las secuencias didácticas se fundamentan en teorías de Rico (1995), Rico y Socas (1994), Grupo Azarquiel(2000) , Deulofeu(1998), Castro E., Rico L., Castro, Enrique, 1996 y otros. 
Las temáticas sobre las que han versado los proyectos en su mayoría pertenecen a los dominios conceptuales del Pensamiento Numérico y Algebraico y las estrategias metodológicas de intervención en el aula están enmarcadas en un enfoque constructivista.: Taller constructivo, Sistemas Concretos, conceptuales y simbólicos, Situaciones didácticas de Brousseau, Modelo de Van Hiele.

> La investigación - acción: una alternativa para la formación inicial de los profesores.

Los cuatro momentos de la Investigación-Acción: exploración y reflexión, planificación, acción y observación, y evaluación están incorporados en la metodología de la asignatura, como estrategia para la construcción y desarrollo del conocimiento práctico profesional.

Con la investigación - acción el estudiante crea hábitos de reflexión sobre la acción desde referentes teóricos espeć́ficos para modificar y hacer evolucionar las concepciones y creencias iniciales sobre las matemáticas, su enseñanza y aprendizaje, con el fin de buscar el mejoramiento continuo de sus prácticas educativas.

\section{$>$ Los proyectos de aula : entorno para la construcción del conocimiento profesional}

Uno de los propósitos de los proyectos de aula en términos de Llinares (1994) es proporcionar entomos de aprendizaje adecuados para facilitar el proceso de construcción del conocimiento profesional, el aual se logra implicando a los estudiantes- profesores en la formulación y resolución de problemas prácticos profesionales, relevantes para su futura labor docente y con sentido para ellos.

La construcción del conocimiento profesional del profesor de matemáticas en formación se lleva a cabo en las dimensiones teórica, práctica e investigativa. La dimensión teórica está presente en el estudio y aplicación de referentes teóricos sobre enrores y dificultades en el aprendizaje de las matemáticas y de propuestas didácticas reportadas en libros y revistas de investigación e innovación en Educación matemática. También se recurre a análisis epistemológicos, didácticos y cognitivos de los objetos matemáticos, en el momento de realizar la transposición didáctica de la matemática disciplinar a la matemática escolar.

En la dimensión práctica sobresalen avances en los procesos de planificación tanto de la investigación en el aula como de la enseñanza, el aprendizaje y la evaluación. Se evidencian conocimientos y competencias profesionales en el diseño y gestión de Unidades y Secuencias didácticas. En cuanto a la forma de abordar los curríaulos de Educación Básica Secundaria y Media se ha pasado del énfasis en contenidos matemáticos al desarrollo de competencias matemáticas. En este proceso se detecta la dificultad de rupturar las creencias y concepciones tradicionales acerca de la enseñanza y aprendizaje de las matemáticas, producto de la experiencia vivida como estudiantes de matemáticas, lo aul se convierte en un factor que obstaculiza el desarrollo del conocimiento profesional.

La dimensión investigativa, se orienta hacia el desarrollo de competencias propias de la investigación en Educación Matemática. Se trata de reconocer al profesor como un investigador de su propia práctica, en donde: el conocimiento disciplinar y su naturaleza, el conocimiento escolar, la enseñanza, el aprendizaje, la evaluación, la transposición didáctica, las relaciones didácticas, etc., se convierten en objetos de estudio, para hacer evolucionar el conocimiento profesional. 


\section{Conclusiones}

Se ha realizado la descripción e interpretación del proceso formativo, que se está llevando a cabo en la asignatura Proyecto Pedagógico Fase I a través del estado del arte de los informes presentados por estudiantes la Licenciatura en Matemáticas y Estadística de la Universidad Pedagógica y Tecnológica de Colombia, Duitama. Se han reconociendo avances y limitaciones, que indican lo que se debe mantener o cambiar para mejorar la formación inicial de los profesores de matemáticas.

Los primeros beneficiados son lo estudiantes de la licenciatura quienes han tenido oportunidad de confrontar la teoría de Didáctica de las Matemáticas en la práctica educativa y crear hábitos de reflexión crítica y sistemática que les permitirá mejorar continuamente su desempeño profesional. En segunda instancia, los proyectos han beneficiado a un buen número de estudiantes de Educación Básica secundaria de seis instituciones educativas del municipio de Duitama.

Se han determinado avances al reconocer la importancia de promover y crear una actitud reflexiva y cuítica sobre las prácticas educativas para mejorarlas continuamente. Estamos de acuerdo con Flores, P. (2004) en que existen diversos momentos, tipos y niveles de reflexión que podemos identificar en la actuación, y que hay que planificar, ejercitar y promover en la formación inicial de profesores.

La metodología de investigación - acción para desarrollar los proyectos de investigación en el aula, a su vez se puede convertir en una altemativa para la formación inicial de profesores.

Otras fortalezas se relacionan con el desarrollo de competencias profesionales relativas a la planificación y gestión de los procesos educativos, la comunicación en el aula, la exploración de la matemática escolar y la percepción de lo que será su profesión futura. Pero, surgen interrogantes como: ces suficiente con esta experiencia de inmersión en la profesión docente y de actuación semiautónoma del estudiante para construir y desarrollar su conocimiento profesional?, ¿Cómo evidenciar la evolución de este conocimiento?, ¿Cuáles son las estrategias más idóneas para promover la construcción de este conocimiento profesional?, etc.

Interrogantes como estos y otras situaciones, pueden constituirse en fuente de investigaciones ayyo desarrollo podńan proporcionar aportes para aualificar los programas de formación inicial y permanente de los profesores de matemáticas.

\section{Bibliografía}

Azcárate, P. y Cardeñoso, J. Ma. (1998). La formación inicial de profesores de matemáticas, finalidades, limitaciones y obstáculos. Investigación en la escuela, № 35, 75-85

Blanco, L. (1998). Nuevos retos en la formación de los profesores de matemáticas. XII Reunión latinoamericana de matemática educativa RELME. Colombia.

Bohórquez, 0.(2008). Los proyectos pedagógicos fase I del programa de licenciatura en matemáticas y estadística: una aproximación al estado del arte. Trabajo de grado. Duitama: Universidad Pedagógica y tecnológica de Colombia.

Font, V. (2005). Matemáticas y su didáctica en la formación inicial. En P. Rojas (Ed.) Memorias XXI Coloquio distrital de matemáticas y estadística-Tomo I (pp. 9-58). Bogotá: Grupo Editorial Gaia.

Flores, P. (2004) Relación con el conocimiento profesional en la formación inicial de profesores de Matemáticas de Secundaria: Reflexión sobre cuestiones profesionales. Memorias de la Sociedad Española de Investigación en Educación Matemática. España: Universidad de Granada

Linares, S. (2005). Aprendizaje del profesor y estrategias de formación. Características de una agenda de investigación. Actas del VII Simposio de la SEIM España: Universidad de Alicante. 